\title{
Intravenous proton pump inhibitors prior to endoscopy in suspected upper gastrointestinal bleeding
}

\section{Clinical question}

Does the use of intravenous proton pump inhibitors (IV PPIs) reduce important adverse outcomes in patients who present to the ED with evidence of acute upper gastrointestinal bleeding?

\section{Article chosen}

Lau JY, Leung WK, Wu JC, et al. Omeprazole before endoscopy in patients with gastrointestinal bleeding. N Engl J Med 2007;356:1631-40.

\section{Objective}

To determine whether early intravenous infusion of a highdose IV PPI before endoscopy would have a therapeutic effect on bleeding ulcers by reducing the need for endoscopic therapy and result in improved clinical outcomes.

\section{Background}

Upper gastrointestinal bleeding is a common presentation to the emergency department (ED) and can require intensive treatment and resources. Management is often carried out in concert with gastroenterologists, who perform diagnostic and therapeutic endoscopy, and rarely with surgeons, who manage patients whose bleeding cannot be controlled with medical therapy. The use of IV PPIs is well supported in the scientific literature for use in upper gastrointestinal bleeds (UGIBs) demonstrating high-risk stigmata at the time of endoscopy. ${ }^{1}$ In a Cochrane systematic review, Leontiadis and colleagues amassed 24 randomized controlled trials with over 4300 patients to show that PPIs are associated with a reduced risk of rebleeding or the need for surgical intervention in patients with UGIBs related to peptic ulcer disease. Selective analyses suggest that in the
Reviewed by: Eddy S. Lang, MDCM, CSPQ; on behalf of the McGill Emergency Medicine Journal Club Group From the Emergency Department, Sir Mortimer B. Davis Jewish General Hospital, McGill University, Montréal, Que.

Received: Oct. 12, 2007; accepted: Nov. 9, 2007

This article has not been peer reviewed.

CJEM 2008; 10(3):244-6

setting of high-risk lesions at endoscopy (i.e., active bleeding, nonbleeding visible vessel) and for studies performed in Asia, there is even an effect on all cause mortality. ${ }^{1}$ Emergency physicians often encounter patients with UGIBs who may experience significant delays before undergoing endoscopy. Furthermore, one expects that only a small proportion of all patients with UGIBs will have highrisk lesions on endoscopy that would mandate the use of IV PPIs. This raises uncertainty as to whether it is beneficial to institute IV PPIs in all patients with suspected UGIBs before they undergo endoscopy.

\section{Population studied}

Consecutive patients who presented with overt signs of UGIB (i.e., melena or hematemesis with or without hypotension) to the Accident and Emergency Department at the Prince of Wales Hospital in Hong Kong were screened for inclusion. Patients with shock (systolic blood pressure $\leq 90 \mathrm{~mm} \mathrm{Hg}$ or pulse $\geq 110$ beats/min) were only considered for entry if they had been stabilized. Patients with continued shock despite initial volume resuscitation (refractory shock) underwent urgent endoscopy and were excluded. Other excluded patients were under 18 years of age, those who were unable to provide written informed consent, pregnant patients and those with a known allergy to PPIs as well as those who were using acetylsalicylic acid (ASA) regularly for cardiovascular protection. Long-term 
ASA users were enrolled in another randomized study, which evaluated the effect of early reintroduction of ASA on the risk of recurrent ulcer bleeding.

\section{Study design}

The study was a prospective randomized-controlled trial with concealed allocation and adequate blinding at multiple levels including assessors of outcome. Patients were randomly assigned to receive an intravenous infusion of omeprazole or placebo, each given as an 80 -mg intravenous bolus injection followed by continuous infusion of $8 \mathrm{mg}$ per hour until endoscopy was performed the next morning. The mean time from study drug initiation to endoscopy was approximately 15 hours. At endoscopy, gastroduodenal ulcers with spurting hemorrhage, oozing hemorrhage or nonbleeding visible vessels were injected with epinephrine followed by coaptive thermocoagulation. Omeprazole $(8 \mathrm{mg} / \mathrm{h})$ was infused for 72 hours after endoscopy in patients who required ulcer hemostasis. Bleeding was considered to have recurred if any of the following occurred: vomiting of fresh blood, hypotensive shock (defined as a systolic blood pressure $\leq 90 \mathrm{~mm} \mathrm{Hg}$ or pulse $\geq 110$ beats/min) with melena after stabilization, or a decrease in the hemoglobin level of more than $20 \mathrm{~g} / \mathrm{L}$ and a decrease in the hematocrit of more than $6 \%$ within 24 hours after a transfusion resulting in a hemoglobin level of $100 \mathrm{~g} / \mathrm{L}$ or less. Patients were followed until day 30 after randomization.

\section{Outcomes measured}

The primary end point of the study was the need for endoscopic therapy at the first endoscopic examination. Secondary end points included signs of bleeding, need for urgent endoscopy, duration of hospital stay, need for transfusion, need for emergency surgery to achieve hemostasis, and rates of recurrent bleeding and death from any cause within 30 days after randomization.

\section{Results}

There were a total of 1511 patients assessed for eligibility over a 17-month period, of which 638 met the inclusion criteria. Three hundred and nineteen patients were randomized into each arm of the trial. Seven patients ( 5 in the IV PPI group and 2 in the placebo group) were excluded from analysis: 3 withdrew before IV PPI or placebo was administered and 4 had received a misdiagnosis of UGIB (2 actually had small-bowel obstruction, one had undergone a total gastrectomy and one had cholangitis). Two patients in the omeprazole group did not undergo endoscopy (1 refused and 1 became moribund). Of the 314 patients in the omeprazole group, $60(19.1 \%)$ required endoscopic treatment, compared with 90 of the 317 patients $(28.4 \%)$ in the placebo group (number needed to treat [NNT] 11, 95\% confidence interval [CI] 8-36). Among patients with peptic ulcer bleeding observed during the first endoscopic examination, actively bleeding peptic ulcers were seen less frequently in patients given omeprazole before endoscopy than in those given placebo (12 of 187 [6.4\%] v. 28 of 190 [14.7\%], respectively; NNT 13, 95\% CI 9-40). The hospital stay was less than 3 days for 190 patients $(60.5 \%)$ in the omeprazole group and 156 patients $(49.2 \%)$ in the placebo group (NNT 9, 95\% CI 5-30). There were no statistically significant differences in the rates of rebleeding or surgical intervention.

\section{Study conclusions}

Infusion of high-dose omeprazole before endoscopy accelerated the resolution of signs of bleeding in ulcers and reduced the need for endoscopic therapy. However, there were no important differences noted in rates of rebleeding, need for surgical intervention or transfusion therapy.

\section{Commentary}

The trial by Lau and colleagues fulfilled all of the methodological criteria for a high-quality randomized controlled trial. Specifically, the researchers employed excellent blinding procedures and there was no loss to follow-up. There were, however, more patients taking ASA and warfarin in the placebo arm and this might have created a bias for the effect of IV PPIs on the primary outcome; but, numbers were small (6 warfarin- and 3 ASA-taking patients) and thus not likely to be significant. The study was also not entirely faithful to the "intention to treat" principle, but the few patients excluded probably did not bias the results. The selection of the need for endoscopic treatment can be considered as surrogate for more patient-important end points as the study was underpowered for rebleeding and the need for surgery.

The results of this study are in keeping with the Cochrane review on IV PPI before endoscopy, with 5 trials and 1500 patients, not including the work by Lau and colleagues reviewed here. ${ }^{2}$ This Cochrane review suggests that early IV PPIs reduces the likelihood of encountering high-risk lesions in need of endoscopic therapy and thus prolonged IV PPI infusions. In keeping with Lau and coauthors, the Cochrane review failed to demonstrate an 
effect on mortality, transfusions, rebleeding or the need for surgical intervention.

Canadian EDs use pantoprozole, which is unlikely to work differently than omeprazole, thus representing a class effect. While not inexpensive (approximately $\$ 75$ for $24 \mathrm{~h}$ of therapy), IV PPIs are safe in the short term and are likely to be cost-effective if they can reduce length of stay, the need for hospitalization and the need for monitored beds in an intensive care unit setting. Thus the early initiation of IV PPIs might have the benefit of reducing hospital stays in patients who would have ordinarily been committed to longer stays (i.e., 72-h infusions) after endocopy. Timely endoscopy is the mainstay of management for ED patients with UGIBs and this study should not be misinterpreted to justify delays in obtaining this diagnostic and often therapeutic intervention.

An important applicability consideration of the work by Lau and colleagues relates to the ethnodemographic dimension of IV PPIs in the setting of UGIBs. Post-hoc work from the Cochrane collaboration suggests an enhanced benefit of IV PPIs in studies conducted in Asian settings and hence, presumably an Asian population..$^{3}$ In this regard, it is reasonable to question whether the results of this trial would be reproducible in a North American context.

Competing interests: None declared.

\section{Conclusion}

Although early use of IV PPIs may only provide a substantial benefit to a small group of ED patients with UGIBs, it is reasonable to embark on this therapy for most patients who require endoscopy during their stay in the ED. Patients with high-risk clinical features and those who experience unavoidable delays before endoscopy may derive the greatest benefit.

\section{References}

1. Leontiadis GI, Sharma VK, Howden CW. Proton pump inhibitor treatment for acute peptic ulcer bleeding. Cochrane Database Syst Rev 2006;1:CD002094.

2. Dorward S, Sreedharan A, Leontiadis GI, et al. Proton pump inhibitor treatment initiated prior to endoscopic diagnosis in upper gastrointestinal bleeding. Cochrane Database Syst Rev 2006;4:CD005415.

3. Leontiadis GI, Sharma VK, Howden CW. Systematic review and meta-analysis: enhanced efficacy of proton-pump inhibitor therapy for peptic ulcer bleeding in Asia - a post hoc analysis from the Cochrane Collaboration. Aliment Pharmacol Ther 2005;21:1055-61.

Correspondence to: Dr. Eddy S. Lang, Emergency Department, SMBD Jewish General Hospital, 3755 Cote Ste. Catherine, Montréal QC H3T 1E2; eddy.lang@mcgill.ca 